\title{
RECENT RESULTS FROM THE OPERA EXPERIMENT
}

\author{
P. DEL AMO SANCHEZ on behalf of the OPERA Collaboration \\ LAPP, 9, Chemin du Bellevue, BP 110 \\ 74941 Annecy-le-Vieux, FRANCE
}

\begin{abstract}
The OPERA neutrino experiment recently finished data-taking, its recorded sample comprising $18.0 \times 10^{19}$ POT delivered by the CERN CNGS beam from 2008 to 2012 . The goal of the OPERA experiment is to establish $\nu_{\mu} \rightarrow \nu_{\tau}$ oscillations in appearance mode by observing the $\tau$ leptons produced in $\nu_{\tau}$ Charged Current interactions. Here we report on the status of the data analysis, and describe, in particular, two $\nu_{\mu} \rightarrow \nu_{\tau}$ candidate events. Results on $\nu_{\mu} \rightarrow \nu_{e}$ oscillations are also presented.
\end{abstract}

\section{Introduction}

Neutrino oscillations have been well established experimentally by neutrino disappearance results $^{1}$. In addition to neutrino disappearance, the oscillation framework also predicts the appearance of neutrinos of different flavours. Conclusive observation of such phenomenon is lacking in the atmospheric sector. In particular, the explanation of the atmospheric $\nu_{\mu}$ disappearance observed by SuperKamiokande ${ }^{2}$, namely that the atmospheric $\nu_{\mu}$ oscillate into $\nu_{\tau}$, since no $\nu_{\mu} \rightarrow \nu_{e}$ excess is seen, is worth testing. OPERA addresses both questions by aiming to observe $\nu_{\tau}$ appearance in a nearly pure $\nu_{\mu}$ beam.

\section{The OPERA experiment}

\subsection{The CNGS beam and the LNGS laboratory}

OPERA is located in the INFN Gran Sasso underground laboratory (LNGS), where it is shielded from cosmic rays by $3800 \mathrm{~m}$ of water equivalent of rock overburden. It has been exposed to the CERN Neutrino to Gran Sasso (CNGS) beam. This gives a baseline of $730 \mathrm{~km}$. The mean neutrino energy, $17 \mathrm{GeV}$, is a compromise between the increase of the $\nu_{\tau}$ Charged Current (CC) interaction cross section at higher energies and the $\nu_{\mu} \rightarrow \nu_{\tau}$ oscillation probability, which, for the considered baseline favours lower energies. The two contradicting requirements imply a mean oscillation probability of only $P\left(\nu_{\mu} \rightarrow \nu_{\tau}\right) \approx 1.7 \%$, a consequence of OPERA not being on an oscillation maximum. The beam is an almost pure $\nu_{\mu}$ beam, the sum of the $\bar{\nu}_{\mu}, \nu_{e}$ and $\bar{\nu}_{e}$ interactions in the detector amounting to less than $3 \%$ of the total. The prompt $\nu_{\tau}$ component of the beam is negligible.

Between 2008 and 2012, the CNGS beam system delivered $18.0 \times 10^{19}$ protons on target $(\mathrm{POT})$.

\subsection{Detection principle}

The appearance of $\nu_{\tau}$ is revealed by the production of $\tau$ leptons, which happens by means of CC interactions during the scattering of the neutrinos off the OPERA target nuclei. Several hadronic 
tracks are often also produced in primary interactions. Due to the high mean neutrino energy and the $\tau$ lifetime, the $\tau$ lepton travels about $1 \mathrm{~mm}$ before decaying. Together with the fact that it decays nearly $85 \%$ of the times to 1-prong final states, the typical $\nu_{\tau}$ experimental signature consists of a track with a kink coming out of the interaction vertex with several hadronic tracks attached. In contrast, the dominant $\nu_{\mu}$ interactions in the target, whether they are Charged or Neutral Current interactions, often produce a vertex of hadronic tracks, sometimes partnered (CC events) by a penetrating track (the muon).

Efficient detection of $\tau$ leptons and good separation of $\nu_{\tau}$ events from $\nu_{\mu}$ events require an excellent spatial resolution. Furthermore, the need of a large target mass to compensate for the small neutrino interaction cross section leads to the so-called Emulsion Cloud Chamber (ECC) concept. In OPERA, it has been implemented in the form of 57 nuclear emulsion films interleaved with 56 1-mm thick $\mathrm{Pb}$ plates, the nuclear emulsion films providing the submicrometric resolution needed. Such ensemble constitutes an OPERA "brick". In addition to the lead-emulsion target, electronic detectors are used to trigger, locate the brick containing the interaction vertex, and measure the properties of penetrating tracks.

\subsection{The OPERA detector}

An OPERA brick weighs $8.3 \mathrm{~kg}$ and corresponds to about 10 radiation lengths. The momenta of the tracks below $8 \mathrm{GeV} / \mathrm{c}^{a}$ are measured thanks to their Multiple Coulomb Scattering in the lead ${ }^{3}$. The excellent angular resolution achieved by the nuclear emulsions $(\sim 2 \mathrm{mrad})$ allows to measure the dispersion of the angle of the track in each emulsion with respect to the global direction of the track in the brick. This dispersion is closely related to the momentum of the particle $^{1}$.

The OPERA detector also has some particle ID capabilities. Nuclear fragments are readily identified by the higher grain density along their tracks in the emulsion, a quantity related to $d E / d x^{4}$. Electron tracks are identified thanks to the showers they originate. Muons are selected with a $95 \%$ efficiency by choosing appropriately long tracks that stretch over the whole detector $^{5}$. When muon identification is essential to validate oscillation candidate events, tracks that fail the previous requirement are followed through the downstream bricks till they either exit the detector or interact. The study of their end-point allows to recover $30 \%$ of the muons missed by the first algorithm ${ }^{4}$.

The OPERA target, with a total mass of $1.25 \mathrm{kton}$, is composed of about 150000 bricks, arranged in walls interleaved with plastic scintillator strips. The latter constitute the Target Tracker (TT) and locate the brick where the interaction has taken place. An equally important function of the TT is muon identification, related to the tracking capabilities it provides for the most penetrating tracks. Last but not least, the TT also triggers the events.

Downstream of the target section, that comprises the brick walls and the TT, there is a muon spectrometer, composed of a dipole magnet generating a field of $1.53 \mathrm{~T}$, and instrumented by RPCs and drift tubes. It measures the charge of the penetrating tracks with a misidentfication probability of $0.3 \%$, and the momentum of those tracks with a precision better than $20 \%$.

Two additional removable emulsion films, called Changeable Sheets (CS), are attached on the downstream face of each brick. When a brick is located by the TT, and extracted by the Brick Manipulating System (BMS) automaton, tracks compatible with the TT hits are searched for in the CS. The spatial resolution of the TT predictions is about $1 \mathrm{~cm}$, whereas the angular resolution is around $25 \mathrm{mrad}$. If tracks corresponding to the TT predictions are found in the $\mathrm{CS}$, the rest of the emulsion films of the brick are developed and sent to one of the scanning laboratories in Europe or Japan, where high speed automated microscopes will scan them. An important step of the data acquisition is done at this stage, when the tracks and vertices recorded in the emulsion are digitally reconstructed.

\footnotetext{
${ }^{a}$ Larger momenta can be measured in a similar fashion, but depend strongly on the local quality of the measurements (angular resolution, density of emulsion black grains unrelated to tracks).
} 
Table 1: Status of data analysis.

\begin{tabular}{|c|c|c|c|}
\hline Years & POT $\left(\times 10^{19}\right)$ & Status & Number of analysed events \\
\hline $2008 \& 2009$ & 5.27 & Completed & 2783 \\
\hline $2010,2011 \& 2012$ & 12.7 & In progress $(\sim 26 \%)$ & 1722 \\
\hline
\end{tabular}

A more complete description of the OPERA detector can be found elsewhere ${ }^{6}$.

\subsection{Backgrounds}

A charm quark is produced in about $4 \%$ of the $\nu_{\mu}$ CC interactions. Since charmed particles have lifetimes that are very similar to that of $\tau$ leptons, whenever the primary muon is not detected or misidentified, the one-prong decays of charm mimic the sought $\tau$ decay kink topology, constituting a background to the $\nu_{\tau}$ oscillation signal in all the channels. A particular background to the $\tau^{-} \rightarrow \mu^{-}$channel is the large angle Coulomb scattering of muons coming from $\nu_{\mu} \mathrm{CC}$ interactions. Finally, a source of background affecting the $\tau^{-} \rightarrow h^{-}$and $\tau^{-} \rightarrow h^{-} h^{+} h^{-}$channels, is the hadronic re-interaction background. Hadrons coming from $\nu_{\mu}$ interactions scatter hadronically off nuclei (for $\mathrm{NC}$ events ${ }^{b}$, it happens $0.2 \%$ of the times), and sometimes result in a kink-like topology.

Some of these backgrounds are suppressed by specific instruments of the detector. For instance, the charm contribution to the $\tau^{-} \rightarrow \mu^{-}$channel consists of wrong-sign muons, which are readily discarded by the muon spectrometer. Most backgrounds, however, are fought using kinematic variables, such as the flight length of the $\tau$ candidate, the missing $p_{T}$ at the primary vertex or the $\phi$ angle. The latter, a powerful discriminating variable, is defined as the angle in the transverse plane between the $\tau$ candidate and the hadronic shower. A true $\tau$ will be back-to-back with respect to the hadronic shower, whereas the angle will be small for fake $\tau$ candidates coming from charm or hadronic reinteractions.

\section{Charm sample}

The decay topologies and lifetimes of charm events, similar to those of the $\tau$ signal, make them an ideal control sample. The agreement of the most important variables between Monte Carlo simulations (MC) and the available data (see Table 1) has been checked, and found to be good (see Figure 1). This proves that no major problems exist in the reconstruction of the relevant variables for OPERA within their ranges of interest, the examples given being the flight length, the $\phi$ angle and the impact parameter of tracks with respect to their vertices of interaction.

A comparison by topology of the expected numbers of events from $\mathrm{MC}$ and the observed numbers of events in data also shows a good agreement (see Table 2).

\section{$4 \quad \nu_{\mu} \rightarrow \nu_{\tau}$ oscillations}

We report here the current results of the $\nu_{\mu} \rightarrow \nu_{\tau}$ oscillation search. The number of expected $\nu_{\tau}$ candidates on the data sample analysed so far (see Table 1) is 1.91 signal events with a background of 0.18 events.

A first $\nu_{\tau}$ candidate was reported in $2010^{7}$. The $\nu_{\tau}$ candidate is consistent with the appearance of a $\tau^{-}$lepton in the OPERA target, that decays through the channel $\tau^{-} \rightarrow \rho^{-} \nu_{\tau}$ with $\rho^{-} \rightarrow \pi^{-} \pi^{0}$ and $\pi^{0} \rightarrow \gamma \gamma$. The tau candidate track exhibits a kink with an angle of

\footnotetext{
${ }^{b}$ The CC contribution to the hadronic background is less important since the probability of not detecting the muon is small $(\sim 5 \%)$.
} 
Table 2: Comparison by topology of numbers of charm events observed in data and MC expectations.

\begin{tabular}{|c|c|c|}
\hline Topology & Expected from MC & Observed in data \\
\hline 1-prong & 28.5 & 19 \\
\hline 2-prong & 22.1 & 22 \\
\hline 3-prong & 8.2 & 5 \\
\hline 4-prong & 1.2 & 4 \\
\hline Total & $60 \pm 8$ & 50 \\
\hline
\end{tabular}
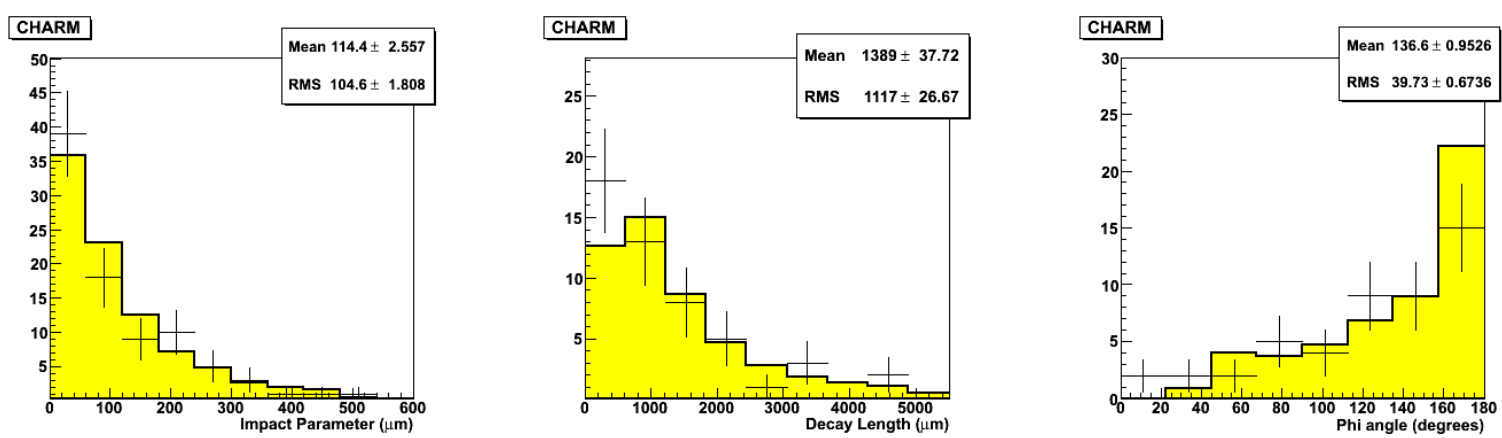

Figure 1: Comparison of charm data (dots with error bars) and MC distributions (yellow histograms) of the impact parameter (left), the decay length (centre) and the $\phi$ angle (right).

$41 \pm 2 \mathrm{mrad}$. The kink is interpreted as arising from a $\tau$ lepton decaying into a 1-prong channel. Its decay length is $1.335 \pm 0.035 \mathrm{~mm}$. The daughter track is identified as a hadron thanks to its interaction 7 walls downstream of the $\tau$ decay point. The two observed photons are consistent with pointing back to the kink, and their invariant mass is compatible that of a neutral pion, $m_{\gamma \gamma}=120 \pm 20$ (stat) \pm 35 (syst). A dedicated scan of the emulsions around the kink did not detect the nuclear fragments that often partner nuclear interactions. The measured momenta of all the tracks lead to a value of $\phi=(173 \pm 3)^{\circ}$, in very good agreement with the expectations, as are the rest of the discriminating variables.

A second $\nu_{\tau}$ candidate was announced at the Neutrino 2012 conference $^{8}$, compatible with a $\tau$ decaying into 3 charged hadrons. This decay channel is not as clean as a typical $\tau \rightarrow 1$ prong topology, with the signal yield being 3 times smaller than in the $\tau^{-} \rightarrow h^{-}$channel and the backgrounds being just as high. The fact that the decay of the $\tau$ candidate happens in the plastic base of the emulsion film, just between the two emulsion layers, helps reducing the probability of the event being a hadronic reinteraction. Indeed, no highly ionizing tracks emitted in nuclear processes (see above) have been found in the emulsions close to the decay vertex. The ranges of the three decay daughters are consistent with the hadron hypothesis. The primary track stops after only two brick walls, also in a fashion compatible with that of a hadron (a muon of the same momentum would most likely cross more than 26 brick walls). Finally, the measured decay length is $1.54 \mathrm{~mm}$, and the observed momenta yield $\phi=(167.8 \pm 1.1)^{\circ}$ as well as values of other kinematic variables in line with the expectations for a $\tau$ decay.

With more than half of the data still to be analysed, there is a high probability of finding additional $\nu_{\tau}$ candidates in the future.

\section{$5 \quad \nu_{\mu} \rightarrow \nu_{e}$ oscillations}

A search for $\nu_{\mu} \rightarrow \nu_{e}$ oscillations was performed on the 2008 and 2009 data sample, corresponding to $5.25 \times 10^{19}$ POT. The $\nu_{e}$ energy is estimated from the energy deposited in the Target Tracker planes, in a similar way as it is done for $\mathrm{CC}$ and $\mathrm{NC} \nu_{\mu}$ interactions ${ }^{5}$. 


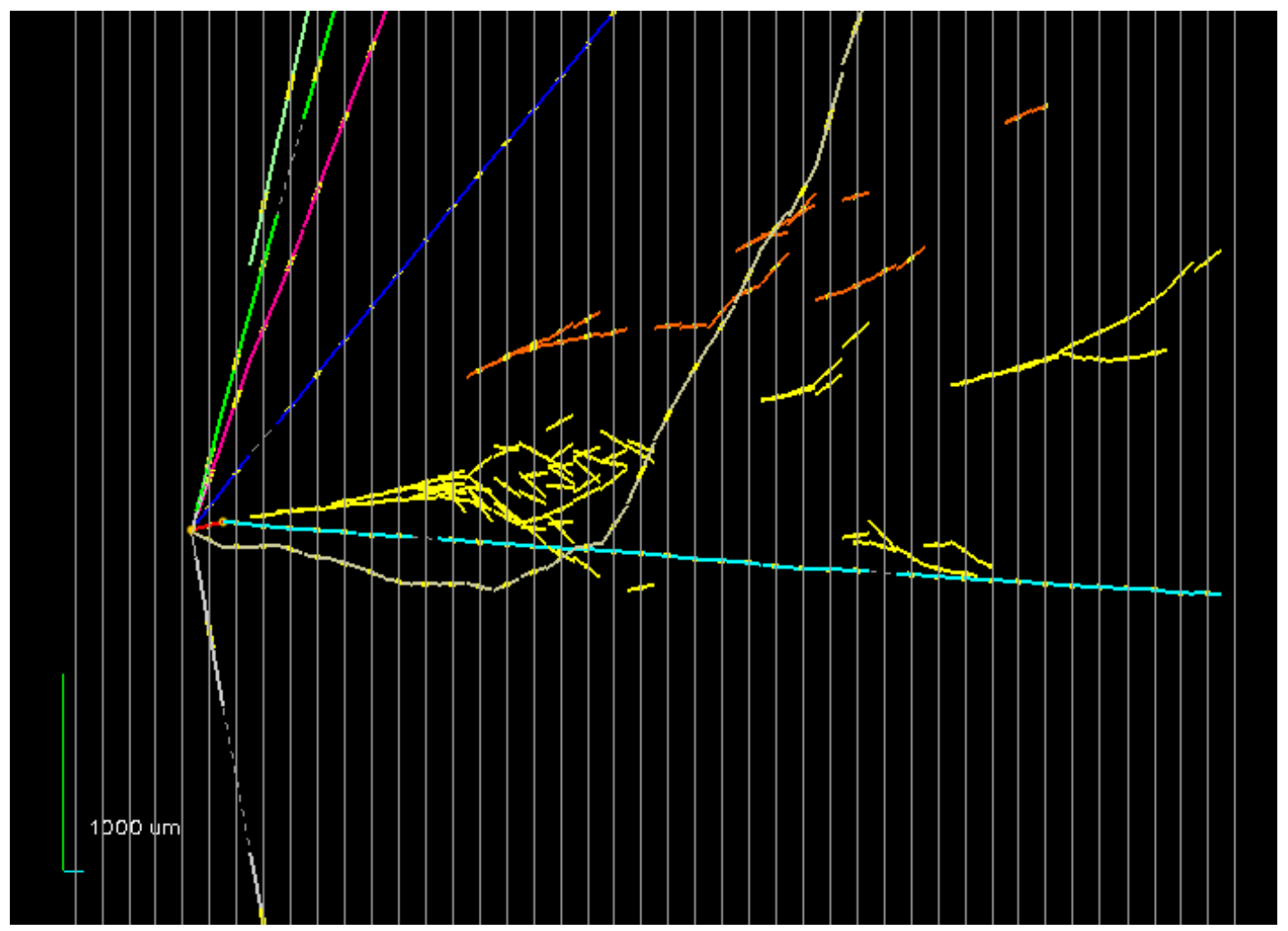

Figure 2: Longitudinal view of the first $\nu_{\tau}$ candidate. The $\tau^{-}$candidate is the short red track attached to the vertex, whereas the $\tau$ daughter is the long green track attached to it. The kink is clearly visible. The two $\gamma$ 's are drawn in yellow and red.

Only 1.4 events are expected from the standard 3-flavour $\nu_{\mu} \rightarrow \nu_{e}$ oscillation for $\sin ^{2}\left(2 \theta_{13}\right)=$ $0.098^{1}$. In contrast, the main background, which arises from beam $\nu_{e}$ contamination, amounts to $19.3 \pm 2.8$ events. A further $0.5 \pm 0.2$ events come from misidentified $\tau \rightarrow e$ and $\pi^{0}$ decays. Whereas the contributions of the first two backgrounds are entirely estimated from simulation, for the latter it is data-driven. The goal is to estimate the probability of a $\gamma$ converting between the interaction vertex and the first emulsion film and mimicking the electron of a $\nu_{e}$ candidate. To that end, the $\nu_{e}$ selection criteria have been applied on a large sample of $\gamma$ 's from data converting several lead plates downstream of the primary interaction. The result is then extrapolated to conversions before the first downstream emulsion film.

A total of 19 events were observed (see Figure 3), in agreement with the expectations, which translates into a rather loose upper limit on $\theta_{13}: \sin ^{2}\left(2 \theta_{13}\right)<0.44$ at $90 \%$ C.L. As expected, OPERA is not able to compete in this matter with other experiments dedicated to this search, and which, contrary to OPERA, are located on an oscillation maximum. However, due to the different $L / E$, OPERA do constrain a different region of the $\sin ^{2}(2 \theta)-\Delta m^{2}$ parameter space. The interest in such constraints has been spurred lately by LSND $^{9}$ and MiniBooNE ${ }^{10}$ results. These experiments have reported possible non-standard $\nu_{\mu} \rightarrow \nu_{e}$ oscillations. These oscillations can be parametrised by an additional contribution given by the standard 2-flavour oscillation formula:

$$
P_{\nu_{\mu} \rightarrow \nu_{e}}=\sin ^{2}\left(2 \theta_{\text {new }}\right) \sin ^{2}\left(1.27 \Delta m_{\text {new }}^{2} L / E\right)
$$

where $\mathrm{L}$ is expressed in $\mathrm{km}$ and $\mathrm{E}$ in $\mathrm{GeV}$. The effects of additional neutrinos are more complex than just adding an extra oscillation term ${ }^{11}$, though, and care must be taken when comparing experiments at different $L / E$.

Thus, considering the standard 3-flavour $\nu_{\mu} \rightarrow \nu_{e}$ oscillation as a background, OPERA excludes the appearance of extra $\nu_{e}$ for certain values of $\sin ^{2}\left(2 \theta_{\text {new }}\right)$ and $\Delta m_{\text {new }}^{2}$ (see Figure 3 ). In particular, we find $\sin ^{2}\left(2 \theta_{\text {new }}\right)<7.2 \times 10^{-3}$ at $90 \%$ C.L. for high values of $\Delta m_{\text {new }}^{2}$. 

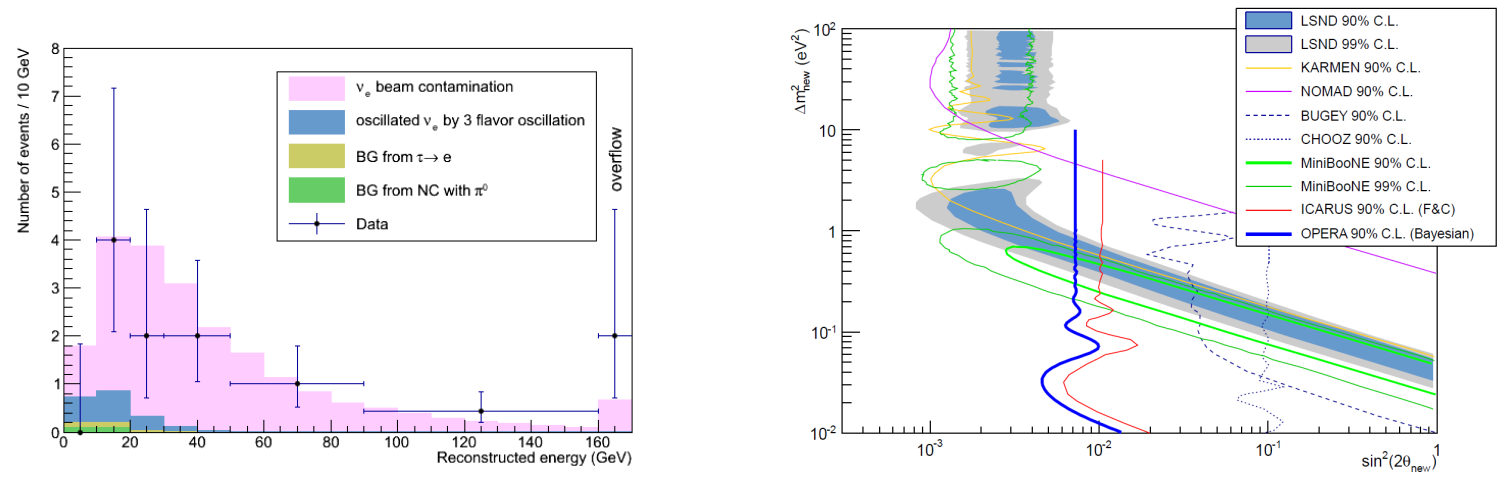

Figure 3: Left, reconstructed $\nu_{e}$ energy spectrum (data points) and expected distributions of the various sources (filled histograms). Right, exclusion plot for the parameters of the non-standard $\nu_{\mu} \rightarrow \nu_{e}$ oscillation; the OPERA $90 \%$ exclusion plot is the continuous blue line.

\section{Conclusions}

The OPERA experiment has successfully recorded events from 2008 to 2012 exploiting the $18.0 \times 10^{19}$ POT delivered by the CNGS beam. The analysis of the data sample is ongoing, about $50 \%$ of it is completed. The search for $\nu_{\mu} \rightarrow \nu_{\tau}$ oscillations has so far identified two $\nu_{\tau}$ candidates, in good agreement with the expectation of 1.9 signal and 0.2 background events. Concerning $\nu_{\mu} \rightarrow \nu_{e}$ appearance, OPERA cannot compete with other experiments due to the fact that its $L / E$ does not correspond to an oscillation maximum. However, OPERA data do constrain interesting parts of the $\Delta m_{\text {new }}^{2}-\sin ^{2}\left(2 \theta_{\text {new }}\right)$ plane, relevant to the possible non-standard $\nu_{\mu} \rightarrow \nu_{e}$ appearance suggested by the LSND and MiniBooNE experiments. In particular, an upper limit $\sin ^{2}\left(2 \theta_{\text {new }}\right)<7.2 \times 10^{-3}$ at $90 \%$ C.L. has been found for high values of $\Delta m_{\text {new }}^{2}$.

\section{References}

1. J. Beringer et al. [Particle Data Group], Phys. Rev. D 86, 010001 (2012)

2. Y. Fukuda et al. [Super-Kamiokande Collaboration], Phys. Rev. Lett. 81, 1562 (1998)

3. N. Agafonova et al. [OPERA Collaboration], New J. Phys. 14, 013026 (2012)

4. N. Agafonova et al. [OPERA Collaboration], New J. Phys. 14, 033017 (2012)

5. N. Agafonova et al. [OPERA Collaboration], New J. Phys. 13, 053051 (2011)

6. R. Acquafredda et al. [OPERA Collaboration], JINST 4, P04018 (2009)

7. N. Agafonova et al. [OPERA Collaboration], Phys. Lett. B 691, 138 (2010)

8. M. Nakamura on behalf of the OPERA collaboration, Results from OPERA, Talk given at the Neutrino 2012 Conference, Kyoto, Japan (2012).

9. A. Aguilar-Arevalo et al. [LSND Collaboration], Phys. Rev. D 64, 112007 (2001)

10. A. A. Aguilar-Arevalo et al. [MiniBooNE Collaboration], arXiv:1207.4809 [hep-ex].

11. B. Bhattacharya, A. M. Thalapillil and C. E. M. Wagner, Phys. Rev. D 85, 073004 (2012) 